\title{
Integration of Multiple Methods in Identifying Patient Safety Risks
}

\author{
M. C. Emre Simsekler ${ }^{\mathrm{a}, \mathrm{b}, \mathrm{c}^{*}}$, Ayse P. Gurses ${ }^{\mathrm{e}, \mathrm{f}, \mathrm{g}}$, Brian E. Smith ${ }^{\mathrm{h}}$, Al Ozonoff $\mathrm{f}^{\mathrm{c}, \mathrm{d}}$
}

a- Khalifa University of Science and Technology, Department of Industrial and Systems Engineering, Abu Dhabi, 127788, UAE

b- University College London, School of Management, London, E14 5AA, UK

c- Division of Infectious Diseases, Department of Medicine, Boston Children's Hospital, 300 Longwood Avenue, Boston, MA, 02115, USA

d- Department of Pediatrics, Harvard Medical School, 25 Shattuck Street, Boston, MA, 02115, USA

e- Armstrong Institute for Patient Safety and Quality, Department of Anesthesia and Critical Care Medicine, Johns Hopkins University School of Medicine, Baltimore, MD, 21287, USA

f- Department of Health Policy and Management, Johns Hopkins University Bloomberg School of Public Health, Baltimore, MD, 21287, USA

g- Johns Hopkins University, Whiting School of Engineering, Baltimore, MD, 21287, USA

h- NASA Ames Research Center, Human Systems Integration Division, Moffett Field, CA, 94035, USA

*Corresponding Author: M. C. Emre Simsekler, Khalifa University of Science and Technology, Department of Industrial and Systems Engineering, P.O. Box 127788, Abu Dhabi, United Arab Emirates, emre.simsekler@ku.ac.ae, T: +971 (0)2 5018410 , F: +971(0)2 4472442

Please cite this work as follows:

Simsekler, M.C.E., Gurses, A.P., Smith, B.E., Ozonoff, A., 2019. Integration of multiple methods in identifying patient safety risks. Saf. Sci. 118, 530-537. https://doi.org/10.1016/j.ssci.2019.05.057

Open access link: https://authors.elsevier.com/a/1ZAwr3IVV9gIPF 


\title{
Integration of Multiple Methods in Identifying Patient Safety Risks
}

\begin{abstract}
There is a growing awareness that risk identification plays an important role in the investigation of actual and potential harm to patients. Although current risk identification methods in healthcare have strengths and limitations, it is an open question whether they have been implemented optimally and how well they have been integrated to provide a complete picture of risk within complex healthcare systems. To shed light on this, this paper reviews the characteristics of reactive and proactive risk identification methods along with their implication on risk identification practices. Various learning points from other safety-critical industries are identified and integration of multiple methods are discussed to provide a more comprehensive view within the scope of risk management. As a particular example, this paper reviews a prognostic method, developed by the Future Aviation Safety Team (FAST), to enhance existing risk identification in the aviation industry by identifying risks that arise due to future changes. The FAST method also demonstrates integration of risk identification methods proposing four complementary approaches for use in the aviation industry. Similarly, our study provides a conceptual framework that can be used in healthcare to integrate multiple methods to accelerate patient safety improvement through comprehensive system coverage. While this paper suggests that such integration may provide better framework for identifying patient safety risks, the low-level maturity of safety management and safety culture should be considered prior to the integration. Future research is also required to provide evidence on effectiveness of integration and relevant costs involved with such integration in healthcare.
\end{abstract}

Keywords: risk identification; systems approach; resilient healthcare, prospective hazard analysis; patient safety; socio-technical systems 


\section{Introduction}

\subsection{Background}

The high rate of medical errors is recognized as a serious problem in healthcare and a threat to patient safety and quality of care around the world. (James, 2013; Makary and Daniel, 2016). The developing movement towards high reliability in healthcare adapts concepts and practices from other safety critical industries such as aviation and nuclear industry (Chassin and Loeb, 2013; Sujan et al., 2018). One key approach to safety across all industries is the investigation of the causal linkage between errors and the risks that underlie those errors. This has brought renewed attention to the identification and mitigation of risks within the scope of risk management as a means for healthcare organizations to reduce errors (Battles and Lilford, 2003; Card et al., 2014).

Risk identification is the core of effective risk management and is necessary to provide a comprehensive view on risks that can occur (Ericson, 2005). The quality of risk assessment depends primarily on thorough risk identification, since mitigation actions cannot be implemented for unidentified risks (Hardy, 2010). Despite this importance, earlier studies show limitations in current risk identification practices (Potts et al., 2014; Simsekler et al., 2015a) and recommend improvements for implementation of these practices in healthcare (Hudson et al., 2012).

One recommendation of note is the integration of current risk identification methods into patient safety programs in complex socio-technical healthcare settings (Sujan and Felici, 2012). Effective action on this recommendation requires a better understanding of the current risk identification practices and characteristics of the methods used so far. This study therefore reviews the current risk identification practice in healthcare. Further it compares healthcare with other safety critical industries with respect to risk identification to learn from their experiences and adopt useful approaches into healthcare settings. In particular, this paper presents lessons from a study (Smith et al., 2008) that evaluated the use of prognostic risk identification method and amalgamation of various tools and methods to provide a comprehensive list of risks within the scope of risk management. 


\subsection{Characteristics of Risk Identification Methods}

There are several definitions and categorisations for basic patient safety and riskrelated terms used in the healthcare practice and research community. There are also ongoing discussions, even confusion, regarding the meaning of such fundamental terms, such as hazard and risk (Habli et al., 2018; Vincent, 2010; Waterson, 2009). While the International Organization for Standardization (ISO) risk management vocabulary guidance (ISO Guide 73, 2009) defines hazard as source of potential harm, risk is defined as the effect of uncertainty on objectives. Similarly, the NHS report (NPSA, 2006) defined hazard as anything that might have the potential to harm, while risk is the consequence of such hazards to patients. Although the definitions above shows the main difference, they are used interchangeably in everyday vocabulary (HSE, 2001).

Similar to the definitions of basic terms, 'hazard identification' and 'risk identification' are also often used interchangeably in the literature. While hazard identification may refer to the identification of the hazard as source of harm (Clarkson et al., 2010; NHS Digital. SCCI0129, 2016), risk identification identifies the hazard as well as cause and effect of the hazard (ISO 31000, 2008; Simsekler et al., 2018a). For instance, risk identification process in the ISO risk management vocabulary guidance (ISO Guide 73, 2009), involves the definition of risk sources, events, their causes, and their potential consequences. As its name suggests, risk identification aims to identify a comprehensive list of risks to be assessed and evaluated regarding their likelihood and severity in risk assessment within the scope of risk management.

There are several methods used for risk identification in both healthcare and other safety-critical industries. While more than 100 methods are available for identifying risks (Gould et al., 2005; Redmill et al., 1999), to date few have been implemented in the patient safety context. Clearly no single method will be suitable for all healthcare settings, and a combination of methods may offer the most effective contribution to risk identification. To this end, we first categorise risk identification methods to provide a useful taxonomy and framework for study:

1. Identifying risks 'after the event' i.e. reactive or retrospective methods 
Recent studies have highlighted current weaknesses in healthcare systems to identify patient safety risks, such as the typically reactive culture with regard to medical error (Braithwaite et al., 2015; Sujan et al., 2017a). This suggests the potential to learn from other industries and scientific disciplines, such as aviation and human factors engineering, which are at the forefront in the development of proactive risk assessment methods (Gurses et al., 2012; Hudson et al., 2012). However, until now few such proactive approaches have been implemented (Simsekler et al., 2015b; Sujan et al., 2018), and difficulties due to different organisational, cultural and regulatory contexts were identified in transition from others to healthcare. For instance, Sujan and his colleagues (Sujan et al., 2016a) reviewed the safety cases for their potential use in healthcare to establish proactive safety management practices and safety performance. However, due to the lower level of maturity of safety management systems in healthcare compared to other traditional safety-critical industries introduced challenges to adopt such tools.

Some have suggested that the healthcare sector should be prudent when selecting and adapting risk identification methods from other industries (Vincent et al., 2012). Organizational aspects associated with effective use of proactive risk identification tools, such as the need for expertise, inputs, and outputs, are highlighted as potential barriers (Ward et al., 2010). Lyons (2009) emphasised lack of practical experience regarding use and selection of proactive risk identification tools in healthcare, and suggested careful guidance when selecting proactive methods due to the diversity and complexity of available tools. Partly in response to these needs and concerns, Clarkson and colleagues (Clarkson et al., 2010) developed the first comprehensive Prospective Hazard Analysis (PHA) healthcare toolkit. Based on a systems approach, the PHA toolkit provides a framework for risk assessment to reduce medical errors, including risk identification, analysis, and evaluation.

An important recommendation of the PHA report is the integration of reactive and proactive methods (Ward et al., 2010). Others have studied linking risk identification with incident reports (Kessels-Habraken et al., 2009). Incident investigation and safety walkabouts are the risk identification techniques most commonly used in healthcare settings (Simsekler et al., 2015b, 2015a; Ward et al., 2010). To date there 
is little research on the integration of such methods in healthcare (Kessels-Habraken et al., 2010, 2009). More frequent use of current methods, together with support and integration of more proactive methods, would likely improve patient safety. Our study aims to understand current practices of reactive methods used in healthcare and to explore integration of proactive methods from other safety-critical industries.

\subsubsection{Reactive methods: identifying risks after an event}

The common idea to all reactive methods is the identification of risks by learning from past experience and failures, which is a key component to focus in traditional safety management (Sujan et al., 2015, 2017b).

Reactive methods share an underlying philosophy that recurrence of errors can be prevented after an incident occurs. The use of such methods might therefore be most acceptable where errors are minor or easily preventable. However, relying solely on these methods is not advisable in settings where errors may result in serious injury, death, or otherwise catastrophic consequences.

Patient safety risk identification has traditionally been reactive in nature within the scope of risk management (Card et al., 2012). While various risk identification methods are available to support patient safety (Shojania, 2012), recent studies showed the most commonly used one is incident reporting and investigation (Hibbert et al., 2018; Kessels-Habraken et al., 2009; Simsekler et al., 2015b). Within this category, patient safety organizations often conduct Root Cause Analysis (RCA) as a primary means of incident investigation (Braithwaite et al., 2006; Iedema et al., 2006; Leistikow et al., 2017; Nicolini et al., 2011a). Compared with the recent emphasis on event reporting (Sujan et al., 2016b), relatively less attention is paid to the thorough and diligent investigation of safety incidents (Vincent, 2004). It should also be noted that in a medical incident investigation that goes into much more in depth than a typical root cause analysis (RCA), the main goal is not necessarily to prevent a similar error or series of errors to occur. The probability that some of these errors will align to create a safety event (following Reason's Swiss Cheese model (Reason, 1990) is extremely low for serious events. The underlying objective of incident investigation should be to improve system safety and to transform organizations into highly reliable environments for care. 
Although incident reporting can be a suitable tool to support risk identification efforts, healthcare organizations have faced challenges with successful implementation (see Table 1). While underreporting is the major limitation, voluntary reporting systems suffer from other limitations including incomplete or inaccurate data; hindsight bias; unreliable classifications; and time delay between an event, its report, the investigation, and the eventual response (Braithwaite et al., 2010, 2008; Spurgeon et al., 2017; Sujan, 2015, 2012; Sujan et al., 2017b; Westbrook et al., 2015). Limited identification of low-level harm, near misses, and potential errors further reduce the value of event reporting systems (Barach and Small, 2000a; Kessels-Habraken et al., 2010). Lack of training and resources for the investigative team is another challenge. Organizations and healthcare staff frequently find difficulty converting knowledge about root causes into appropriate risk treatment plans (Card et al., 2012; Nicolini et al., 2011b).

Even though we have mainly introduced incident reporting and investigation as particular risk identification methods due to their theoretical basis and particular merit, there are also other methods that can feed risk identification process (Woloshynowych et al., 2005). For instance, while comparing healthcare with othersafety critical industries, we should note the effort of healthcare in putting patients at the heart of any improvement. Flott and colleagues (2017) recently studied the potential use of patient-reported feedback to fully integrate their views into their care pathways. Similar to this, Armitage et al. (2018) compared different incident reporting tools and identified that incidents reported by patients are not likely to be identified through other methods. Further, Khan et al. (2016) showed that most errors identified by parents of patients were not in the medical record. Such studies suggested that there is much to be learned from patients, caregivers, and parents about the failures in healthcare systems (Harrison et al., 2015; Lawton et al., 2017; Weingart et al., 2017). Therefore, committee meeting minutes, claims data, survey reports, patient complaints, and standardised patient satisfaction surveys may also be used to feed the risk identification process.

We conclude that incident investigation is indeed useful once an incident has occurred. However, its reactive nature is problematic in terms of risk identification strategies (Hoyle, 2005). Reporting and investigation is more effective if the 
organizational culture supports reporting 'potential' errors, which would in fact make those risk prevention strategies proactive. Incident investigations can provide learning opportunities (Barach and Small, 2000b; Kim et al., 2007; Rollenhagen et al., 2010; Sammer et al., 2010) but do little to inform on causes and prevention of future errors. Investigative methods may not address the full range of potential risks, and event reporting may therefore identify an incomplete list of risks for a given healthcare system (NOPSA, 2011). For these reasons, other safety-critical industries treat reporting methods as supplementary to proactive risk identification systems by providing valuable information on root causes and contributing factors (Hardy, 2010; NOPSA, 2011).

\subsubsection{Proactive methods: predicting risks before an event}

Prospective Hazard Analysis (PHA) methods include standard practices such as Failure Modes and Effects Analysis (FMEA) (van Tilburg et al., 2006), Hazard and Operability (HAZOP) (Lyons et al., 2004), and Structured-What-If Technique (SWIFT) (Card et al., 2012). These tools, afford proactive analysis of risks rather than learning from past errors (Kessels-Habraken et al., 2009; Simsekler and Jayaraman, 2018). There are also additional tools available beyond PHA methods, such as the safety walkabout (Lim et al., 2014) used to identify potential risks before harm occurs in systems.

Compared to healthcare, safety-critical industries have more experience with the use of PHA methods (Sujan et al., 2018). Moreover, proactive risk surveillance is already embedded in the culture of other safety-critical industries, while awareness of the benefits of using these methods has only recently increased in healthcare. For instance, the Joint Commission for Accreditation of Healthcare Organizations (JCAHO) requires accredited organisations to use proactive methods as part of their organisational patient safety policies (Battles and Kanki, 2004; Kessels-Habraken et al., 2009; NPSA, 2006).

Compared to reactive methods, proactive methods do not rely only on past events. Further, they offer significant theoretical benefits over reactive methods. While reactive investigations mainly depend on the incidents reported, PHA methods suggest various tools and techniques to meet specific needs of most analyses 
(Kessels-Habraken et al., 2010; Smith et al., 2010). Further, they are mostly based on systems approaches that can help identify the real causes of patient safety problems (Shojania and Dixon-Woods, 2013).

When comparing the guidance afforded by the use of proactive methods in healthcare and other safety-critical industries, the structure of the overall risk identification process is well defined and more comprehensive in safety-critical industries (ESSI/ECAST, 2009; Eurocontrol, 2004; Harvard Energy, 2009; NOPSA, 2011) compared to healthcare. While incident investigation is the primary risk identification method in healthcare, it is used only as a supplement to the formal proactive methods employed in other safety-critical industries (Harvard Energy, 2009). Incident investigation plays an important role in identifying the causes of failures in other safety-critical industries, but the risk identification process has made further advances.

Although various PHA methods are used for risk assessment in various industries, not all of them encompass every element of the generic risk management process. Because each method has different inherent attributes, its utility necessarily differs from that of other such methods. Other safety-critical industries select and use applicable methods according to their industrial characterisation. Gould et al. (2005) conducted a comprehensive literature review for the Health and Safety Laboratory (An Agency of the Health and Safety Executive, HSE) to examine the applicability of proactive risk identification methods in safety-critical industries. Many of these methods are structured brainstorming techniques that engage stakeholders to minimise risks and promote evidence-based safety. The European Commercial Aviation Safety Team identified more than 40 tools and methods for identifying risks (ESSI/ECAST, 2009). Most such proactive tools are relatively unknown and underutilized in healthcare settings. Further, most PHA methods have not been validated to categorise in which particular healthcare system they would be more useful (Potts et al., 2014).

As briefly introduced earlier, Clarkson and his colleagues developed the PHA Toolkit to support the integration of PHA methods into healthcare (Clarkson et al., 2010). This toolkit identified a number of PHA methods that would be useful in healthcare and determined the extent to which each of these methods is currently used. The research underlying the toolkit gathered and organized otherwise disparate knowledge 
and experience of healthcare workers in applying prospective methods to healthcare delivery. It showed that PHA methods have in fact been little used in healthcare, and the experience of their use in that setting is very rarely described in the literature when compared to other safety-critical industries. The only exception is that of FMEA, where the time-intensive nature of the method was often described as a significant constraint (Simsekler et al., 2019; Ward et al., 2010). Slow adoption of PHA methods has been reported previously (Lyons, 2009; Potts et al., 2014; Simsekler, 2019). It can therefore be suggested that it is essential to choose the most appropriate method for a given system, within available resources.

\section{Integrating Multiple Risk Identification Methods}

The review above highlights the distinct nature of reactive versus proactive risk identification methods. We further compare characteristics of these two approaches in Table 1. These approaches are complementary, and when combined will generate a more comprehensive list of risks than either approach will produce on its own. This would support healthcare organizations' transition from Safety-I to Safety-II, where safety management cannot only be reactive but also proactive (Hollnagel, 2014). Moreover, such integration would potentially provide a platform to merge current safety approaches, which aims to reduce things that go wrong, with resilient health care, which aims to increase and improve the number of things that go right (Braithwaite et al., 2015; Hollnagel, 2014; Sujan et al., 2017b) . A review of safetycritical industries also showed a combination of different methods and approaches (ESSI/ECAST, 2009; NOPSA, 2011) in risk identification.

\section{--- INSERT TABLE-1 ABOUT HERE ---}

The use of complementary approaches is also promoted by NASA (Battles et al., 2006). Although Probabilistic Risk Assessment (PRA) is an accepted safety research tool to study risk in technical systems, its use is limited in predominately sociotechnical systems where human limitations and failure represent substantial risks. There is an increasing awareness in healthcare that triangulation of data from multiple sources and methods is needed for comprehensive risk assessment (Olsen et al., 2007; Sari et al., 2007; Simsekler et al., 2018a). 
Compared to reactive risk methods, PHA methods are generally more open and extensive (Karnon et al., 2006). In theory PHA methods can foresee all potential failures, thus making reactive risk analysis redundant (Kessels-Habraken et al., 2009). Further, empirical evidence suggests proactive approaches identify different sets of risks versus those identified by reactive approaches (Simsekler et al., 2015a).

Although the power of proactive methods is clear, there are important reasons to continue performing retrospective investigation of safety events in healthcare (Vincent, 2006) and there is no sharp division between reactive and proactive methods. Incident reports are used to identify existing limitations and potential problems, while proactive methods identify and assess risks by leveraging the knowledge and experience of frontline workers. This implies that reactive data are used to develop proactive failure scenarios.

Following Vincent et al. (2006), we assume that future risk identification strategies will combine reactive and proactive methods by analysing incident reports to generate hypotheses and establish enthusiasm for more structured proactive methods. This may overcome possible biases in both types of methods. Earlier studies have suggested using reactive and proactive methods in combination to yield a more complete picture of risks. These data and findings would support healthcare leaders to prioritize patient safety and quality resources appropriately (Battles and Lilford, 2003; Hollnagel, 2008; Kessels-Habraken et al., 2009; Senders, 2004; Simsekler et al., 2018b). Moreover, such integrative methods can bring about improvements in the safety research context by improving the methods themselves (Kessels-Habraken et al., 2009).

Box 1:
A proactive hazard identification approach can complement traditional risk
methods to identify a comprehensive list of aviation risks.
The Prognostic Hazard Identification method, developed by the Future
Aviation Safety Team (FAST), focuses on identifying imagined and
unimagined hazards that arise due to concrete, foreseeable future changes in 
the aviation industry (ESSI/ECAST, 2009; Smith et al., 2008). This method has been applied in aviation but not yet in healthcare.

In terms of learning from other safety-critical industries, the FAST method (Smith et al., 2008) identified a number of characteristics of risk identification that can be adapted to healthcare settings.

FAST aims to enhance existing risk identification by predicting future changes internal and external to the aviation industry (Smith et al., 2008). Members of a FAST team are expected to identify change phenomena known as Areas of Change (AOCs) in 11 socio-technical categories relevant to aviation ranging from aircraft technologies to organizational dynamics. Future AOCs may affect today's safety levels and risk within the future system. A number of key questions are applied such as "Does this AoC increase the likelihood of well-understood current hazards that will exist in the future?" By considering the full range of future developments, systematically unanticipated future hazards can be revealed proactively (Smith et al., 2008).

Smith et al. (2008) demonstrated a combination of risk identification methods in FAST categorization, proposing four complementary risk identification approaches for use in the aviation industry:

i. Functional methods such as FMEA;

ii. Methods such as HAZOP to reveal 'functionally unimaginable hazards';

iii. Brainstorming techniques employing "what if" questions; and

iv. Cross-domain, prognostic techniques such as FAST that utilize known future vectors to increase the range and quality of imagined and unimagined hazards.

In this classification, readily imagined hazards are identified by functional methods such as FMEA within the first phase of risk identification. Although FMEA is systematic from a functional failure point of view, there are 'functionally unimagined hazards', or more briefly 'unimagined hazards', which may not be discovered via FMEA. Such hazards can be identified using a second set of tools such as HAZOP (Eurocontrol, 2004; Smith et al., 2008). The advantage of using HAZOP is that unimagined hazards are revealed using guidewords e.g. more, less, before, after, etc. 
Pure brainstorming is a third important risk identification approach that should not be overlooked. Using FAST methods as a fourth approach reveals a rich set of additional hazards. While such a multi-stage prognostic approach has been applied in aviation to identify AoCs, neither equivalent tools, nor methods, nor the same level of rigor has been used in the patient safety context to create an inventory of foreseeable future changes.

There are inevitable overlaps among the hazards identified by each method, and a combination of methods can potentially enhance the risk identification process by increasing the quantity and quality of hazards identified. In addition to methodological triangulation, the adoption within healthcare of FAST or a similar approach can also improve patient safety by considering organizational, contextual, and environmental factors. Frameworks similar to FAST, with new categories specific to healthcare, can be tailored to the patient safety purview.

The current methods used in healthcare, and their potential triangulation, suggest further questions regarding risk identification methodology. What results would be obtained with the current methods, such as incident investigations or safety walkabouts? Can any attempt to triangulate different methods achieve an accurate understanding of system-wide healthcare risks? Early studies offer evidence that none of the current methods should be used in isolation, but instead should be integrated with other tools and methods for a more robust review of the risk environment (Potts et al., 2014; Simsekler et al., 2015a). Results of this review can be represented visually with the help of the learning points from the FAST approach (Smith et al., 2008).

There are classifications similar to FAST that are especially suited to the patient safety context. Frameworks such as SEIPS (Systems Engineering Initiative for Patient Safety) illustrate health system components, error-producing conditions, and potential sources of risks (Carayon et al., 2006; NPSA, 2009; Rogers, 2002; Runciman et al., 2006; Simsekler et al., 2015a; Thomas Diller et al., 2014; Vincent et al., 1998). While most components in such frameworks are broadly similar to those in other safetycritical industries, the following categorization is particularly useful in the context of risk identification for healthcare (Simsekler et al., 2015a): (i) patient-sourced risks; 
(ii) staff-sourced risks; (iii) task-related risks; (iv) environmental risks; ( equipment-related risks; (vi) communication risks; and (vii) organizational risks.

Figure 1 illustrates the conceptual model of learning from the FAST approach to healthcare using the categorization above. In this schematic, a particular healthcare organization has adopted 5 risk identification methods, each of which reveals an important component of the larger risk environment. This is a conceptual schematic and qualitative depiction and thus the sizes and distributions of the ovals are not drawn to scale, nor do we quantify, or correlate risks identified using the different methods. Instead the figure illustrates that different reactive and proactive methods can provide different information about risk. The inevitable overlap across methods offers further opportunities for cross-validation.

\section{--- INSERT FIGURE-1 ABOUT HERE ---}

The figure also suggests that no single method is likely to identify all possible risks; even applying multiple methods may not guarantee identification of all risks across the components of a given system. Therefore, using complementary methods jointly is a more successful risk identification strategy, and no single method should be used as a stand-alone tool to provide a comprehensive list of risks. After combination and integration, reactive and proactive risk identification methods should be guided by a conceptual model that incorporates all system components (Simsekler et al., 2015a). In this way risks can be identified comprehensively and without too narrow a focus on one component at the expense of others.

While there are many expected benefits from using multiple methods in risk identification, there are also some potential challenges that must be addressed. One concern when using a combined approach is the effort and resources needed to implement each method. Since the healthcare field must work within significant time and financial constraints, it is important to consider the trade-off of cost versus utility of the integration of multiple methods. The evidence base for integration of reactive and proactive methods in healthcare is not well established yet. In fact, there is no study from other traditional safety-critical industries showing the implication of integration empirically. Only exception is the risk identification guidance, policy and procedure documents that recommend the idea of integration (ESSI/ECAST, 2009; NOPSA, 
2011). Available studies are only a few from healthcare suggesting that applying a proactive method promotes incident reporting (Kessels-Habraken et al., 2010). Since there are only few studies available (Kessels-Habraken et al., 2010, 2009), the use of multiple inputs in the risk identification process is not well understood yet. Another potential challenge is a lack of risk information exchange across and between different methods and failure to integrate risk information collected from disparate methods. Although risk register systems are expected to aid the amalgamation of risk information, results from early studies have shown limited ability of healthcare organisations to integrate risk information into a single system akin to a 'risk library' (Simsekler et al., 2015a). In aviation, a useful model of such a library has been developed by the International Civil Aviation Organization (ICAO, 2013). Figure 2 shows the adaptation of the ICAO library to integrate clearly a range of healthcare risk identification methods. Risks identified by any of the methods would be captured and categorized appropriately in the risk library. In this figure, documentation refers to putting all risks, identified by various tools and methods, in the same library and categorized depending on their impact (high, moderate, and low) so healthcare organizations can start controlling risks in a structured way regarding their mitigation and treatment plan. Though such a library can be adopted to archive and communicate information about risks to stakeholders in healthcare, practical challenges remain around oversight, implementation, access, and usage.

\section{--- INSERT FIGURE-2 ABOUT HERE ---}

Simply identifying risks, without deep analysis, may have a negative impact on safety culture, leading to bad decisions and ultimately future risks. A well-defined transition between identification and assessment will help healthcare organisations to optimize risk mitigation strategies. Without prudence, applying new tools or integrating multiple methods may result in a risk bureaucracy without contributing in a meaningful way to safety culture. Considering all these recommendations, future research is required to focus on providing empirical evidence on the unique potential contribution of each method to the overall risk identification, and further contribution of the integration of methods to patient safety and risk management. Besides providing evidence on the effectiveness, future research should also analyse the costeffectiveness involved with such an integration. 


\section{Conclusion}

Our study evaluates reactive and proactive risk identification methods; examines their contribution when each is used as a stand-alone method; and considers the implications of integrating across the two domains. Despite scant evidence in the healthcare literature on how such integration should be implemented, experiences of other safety critical industries show that the integration is feasible and likely to provide a more comprehensive identification of risks in any chosen healthcare setting.

Considering issues in the current risk identification practice within the scope of risk management process, integration may open room for improvement. Though such integration should bring a more complete picture of risks, its possible implication on the quality of risks and practicality are potential challenges that can be considered in future research.

It should be noted that not only the idea of integration, but also most proactive tools and methods are still unknown and underused in healthcare. As suggested in earlier studies in particular examples, such as FMEA (Simsekler et al., 2019) and safety cases (Sujan et al., 2016a), healthcare stakeholders require access to guidance and training to be able to select and use tools and methods in an effective way. As noted from four complementary risk identification approaches through FAST in aviation, such guidance should also help healthcare stakeholders and regulatory bodies to better understand the contribution of various risk identification approaches and potential integration. While these should be supported by the regulatory bodies and providers, healthcare stakeholders and safety science researcher should also work in collaboration to provide empirical evidence of the integration of reactive and proactive tools in improving safety management practices, safety culture and safety performance.

\section{Competing Interests}

The authors declared no potential conflicts of interests with respect to the authorship and/or publication of this article. 


\section{References}

Armitage, G., Moore, S., Reynolds, C., Laloë, P.-A., Coulson, C., McEachan, R., Lawton, R., Watt, I., Wright, J., O'Hara, J., 2018. Patient-reported safety incidents as a new source of patient safety data: an exploratory comparative study in an acute hospital in England. J. Health Serv. Res. Policy 23, 36-43. https://doi.org/10.1177/1355819617727563

Barach, P., Small, S.D., 2000a. How the NHS can improve safety and learning. BMJ 320, 1683-1684. https://doi.org/10.1136/bmj.320.7251.1683

Barach, P., Small, S.D., 2000b. Reporting and preventing medical mishaps: lessons from non-medical near miss reporting systems. BMJ 320, 759-763.

Battles, J.B., Dixon, N.M., Borotkanics, R.J., Rabin-Fastmen, B., Kaplan, H.S., 2006. Sensemaking of Patient Safety Risks and Hazards. Health Serv. Res. 41, 1555-1575. https://doi.org/10.1111/j.1475-6773.2006.00565.x

Battles, J.B., Kanki, B.G., 2004. The Use of Socio-Technical Probabilistic Risk As sessment at AHRQ and NASA, in: Probabilistic Safety Assessment and Management. Springer, Berlin, pp. 2212-2217.

Battles, J.B., Lilford, R.J., 2003. Organizing patient safety research to identify risks and hazards. Qual. Saf. Health Care 12, ii2-ii7. https://doi.org/10.1136/qhc.12.suppl_2.ii2

Braithwaite, J., Wears, R.L., Hollnagel, E., 2015. Resilient health care: turning patient safety on its head. Int. J. Qual. Health Care 27, 418-420. https://doi.org/10.1093/intqhe/mzv063

Braithwaite, J., Westbrook, M., Travaglia, J., 2008. Attitudes toward the large-scale implementation of an incident reporting system. Int. J. Qual. Health Care 20, 184191. https://doi.org/10.1093/intqhe/mzn004

Braithwaite, J., Westbrook, M.T., Mallock, N.A., Travaglia, J.F., Iedema, R.A., 2006. Experiences of health professionals who conducted root cause analyses after undergoing a safety improvement programme. BMJ Qual. Saf. 15, 393-399. https://doi.org/10.1136/qshc.2005.017525

Braithwaite, J., Westbrook, M.T., Travaglia, J.F., Hughes, C., 2010. Cultural and associated enablers of, and barriers to, adverse incident reporting. BMJ Qual. Saf. 19, 229-233. https://doi.org/10.1136/qshc.2008.030213

Carayon, P., Schoofs Hundt, A., Karsh, B.-T., Gurses, A.P., Alvarado, C.J., Smith, M., Flatley Brennan, P., 2006. Work system design for patient safety: the SEIPS model. Qual. Saf. Health Care 15 Suppl 1, i50-58. https://doi.org/10.1136/qshc.2005.015842 
Card, A.J., Simsekler, M.C.E., Clark, M., Ward, J.R., Clarkson, P.J., 2014. Use of the Generating Options for Active Risk Control (GO-ARC) Technique can lead to more robust risk control options. Int. J. Risk Saf. Med. 26, 199-211. https://doi.org/10.3233/JRS-140636

Card, Alan J, Ward, J.R., Clarkson, P.J., 2012. Beyond FMEA: the structured what-if technique (SWIFT). J. Healthc. Risk Manag. J. Am. Soc. Healthc. Risk Manag. 31, 23-29. https://doi.org/10.1002/jhrm.20101

Card, Alan J., Ward, J.R., Clarkson, P.J., 2012. Getting to zero: evidence-based healthcare risk management is key. J. Healthc. Risk Manag. J. Am. Soc. Healthc. Risk Manag. 32, 20-27. https://doi.org/10.1002/jhrm.21091

Chassin, M.R., Loeb, J.M., 2013. High-Reliability Health Care: Getting There from Here. Milbank Q. 91, 459-490. https://doi.org/10.1111/1468-0009.12023

Clarkson, P.J., Ward, J.R., Buckle, P., Berman, J., 2010. Prospective Hazard Analysis Toolkit (No. 978- 0-9545243-4- 0). University of Cambridge, Cambridge.

Ericson, C.A., 2005. Hazard Analysis Techniques for System Safety. WileyBlackwell.

ESSI/ECAST, 2009. Guidance on Hazard Identification. ECAST Safety Management System and Safety Culture Working Group.

Eurocontrol, 2004. Guidelines for the Identification of Hazards: How to make unimaginable hazards imaginable? National Aerospace Laboratory NLR, Brussels.

Flott, K.M., Graham, C., Darzi, A., Mayer, E., 2017. Can we use patient-reported feedback to drive change? The challenges of using patient-reported feedback and how they might be addressed. BMJ Qual. Saf. 26, 502-507. https://doi.org/10.1136/bmjqs2016-005223

Gould, J., Glossop, M., Ioannides, A., 2005. Review of Hazard Identification Techniques. Health \& Safety Laboratory (An agency of the Health and Safety Executive)., Sheffield.

Gurses, A.P., Ozok, A.A., Pronovost, P.J., 2012. Time to accelerate integration of human factors and ergonomics in patient safety. BMJ Qual. Saf. 21, 347-351. https://doi.org/10.1136/bmjqs-2011-000421

Habli, I., White, S., Sujan, M., Harrison, S., Ugarte, M., 2018. What is the safety case for health IT? A study of assurance practices in England. Saf. Sci. 110, 324-335. https://doi.org/10.1016/j.ssci.2018.09.001

Hardy, T.L., 2010. Using Accident Report to Improve the Hazard Identification Process. Presented at the 28th International System Safety Conference, Denver.

Harrison, R., Walton, M., Manias, E., Smith-Merry, J., Kelly, P., Iedema, R., Robinson, L., 2015. The missing evidence: a systematic review of patients' 
experiences of adverse events in health care. Int. J. Qual. Health Care J. Int. Soc. Qual. Health Care 27, 424-442. https://doi.org/10.1093/intqhc/mzv075

Harvard Energy, 2009. Harvard Energy Safety Program Manual. OPX Consulting Inc.

Hibbert, P.D., Thomas, M.J.W., Deakin, A., Runciman, W.B., Braithwaite, J., Lomax, S., Prescott, J., Gorrie, G., Szczygielski, A., Surwald, T., Fraser, C., 2018. Are root cause analyses recommendations effective and sustainable? An observational study. Int. J. Qual. Health Care 30, 124-131. https://doi.org/10.1093/intqhc/mzx181

Hollnagel, E., 2014. Safety-I and Safety-II : The Past and Future of Safety Management. CRC Press. https://doi.org/10.1201/9781315607511

Hollnagel, E., 2008. Risk + barriers = safety? Occup. Accid. Scenar. Accid. Anal. Pap. Sel. Third Int. Conf. Work. Saf. WOS2006 Sept. 12-15th 2006 Zeewolde Netherland 46, 221-229. https://doi.org/10.1016/j.ssci.2007.06.028

Hoyle, A., 2005. A Basic Guide to Patient Safety [WWW Document]. URL http://careers.bmj.com/careers/advice/view-article.html?id=1098 (accessed 5.11.13).

HSE, 2001. Reducing risks, protecting people: HSE's decision-making process. Health and Safety Executive, Norwich.

Hudson, D.W., Holzmueller, C.G., Pronovost, P.J., Gianci, S.J., Pate, Z.T., Wahr, J., Heitmiller, E.S., Thompson, D.A., Martinez, E.A., Marsteller, J.A., Gurses, A.P., Lubomski, L.H., Goeschel, C.A., Pham, J.C., 2012. Toward improving patient safety through voluntary peer-to-peer assessment. Am. J. Med. Qual. Off. J. Am. Coll. Med. Qual. 27, 201-209. https://doi.org/10.1177/1062860611421981

ICAO, 2013. Safety Management Manual (SMM). International Civil Aviation Organization, Canada.

Iedema, R.A.M., Jorm, C., Braithwaite, J., Travaglia, J., Lum, M., 2006. A root cause analysis of clinical error: Confronting the disjunction between formal rules and situated clinical activity. Soc. Sci. Med. 63, 1201-1212. https://doi.org/10.1016/j.socscimed.2006.03.035

ISO 31000, 2008. ISO 31000:2009 - Risk management -- Principles and guidelines. The International Organization for Standardization, Geneva.

ISO Guide 73, 2009. ISO Guide 73:2009 Risk management -- Vocabulary. The International Organization for Standardization, Switzerland.

James, J.T., 2013. A New, Evidence-based Estimate of Patient Harms Associated with Hospital Care: J. Patient Saf. https://doi.org/10.1097/PTS.0b013e3182948a69

Karnon, J., McIntosh, A., Bath, P., Dean, J., Hutchinson, A., Oakley, J., FreemanParry, L., Pratt, P., Thomas, N., Karsh, B.-T., Gandhi, T., Tappenden, P., 2006. A 
prospective hazard and improvement analysis of medication errors in a UK secondary care setting. Report for the NHS Patient Safety Programme.

Kessels-Habraken, M., De Jonge, J., Van der Schaaf, T., Rutte, C., 2010. Prospective risk analysis prior to retrospective incident reporting and analysis as a means to enhance incident reporting behaviour: a quasi-experimental field study. Soc. Sci. Med. 1982 70, 1309-1316. https://doi.org/10.1016/j.socscimed.2010.01.035

Kessels-Habraken, M., Van der Schaaf, T., De Jonge, J., Rutte, C., Kerkvliet, K., 2009. Integration of prospective and retrospective methods for risk analysis in hospitals. Int. J. Qual. Health Care J. Int. Soc. Qual. Health Care ISQua 21, 427-432. https://doi.org/10.1093/intqhc/mzp043

Khan, A., Furtak, S.L., Melvin, P., Rogers, J.E., Schuster, M.A., Landrigan, C.P., 2016. Parent-Reported Errors and Adverse Events in Hospitalized Children. JAMA Pediatr. 170, e154608-e154608. https://doi.org/10.1001/jamapediatrics.2015.4608

Kim, J., An, K., Minah Kang Kim, Sook Hee Yoon, 2007. Nurses' Perception of Error Reporting and Patient Safety Culture in Korea. West. J. Nurs. Res. 29, 827-844. https://doi.org/10.1177/0193945906297370

Lawton, R., O’Hara, J.K., Sheard, L., Armitage, G., Cocks, K., Buckley, H., Corbacho, B., Reynolds, C., Marsh, C., Moore, S., Watt, I., Wright, J., 2017. Can patient involvement improve patient safety? A cluster randomised control trial of the Patient Reporting and Action for a Safe Environment (PRASE) intervention. BMJ Qual. Saf. 26, 622-631. https://doi.org/10.1136/bmjqs-2016-005570

Leistikow, I., Mulder, S., Vesseur, J., Robben, P., 2017. Learning from incidents in healthcare: the journey, not the arrival, matters. BMJ Qual. Saf. 26, 252-256. https://doi.org/10.1136/bmjqs-2015-004853

Lim, R.B.T., Benjamin Boon Lui, N., Kok Mun, N., 2014. Evaluation of the Patient Safety Leadership Walkabout programme of a hospital in Singapore. Singapore Med. J. 55, 78-83. https://doi.org/10.11622/smedj.2014019

Lyons, M., 2009. Towards a framework to select techniques for error prediction: supporting novice users in the healthcare sector. Appl. Ergon. 40, 379-395. https://doi.org/10.1016/j.apergo.2008.11.004

Lyons, M., Adams, S., Woloshynowych, M., Vincent, C., 2004. Human reliability analysis in healthcare: A review of techniques. Int. J. Risk Saf. Med. 223-237.

Makary, M.A., Daniel, M., 2016. Medical error - the third leading cause of death in the US. BMJ 353, i2139. https://doi.org/10.1136/bmj.i2139

NHS Digital. SCCI0129, 2016. DCB0129: Clinical Risk Management: its Application in the Manufacture of Health IT Systems. Standardisation Committee for Care Information. 
Nicolini, D., Waring, J., Mengis, J., 2011a. The challenges of undertaking root cause analysis in health care: a qualitative study. J. Health Serv. Res. Policy 16 Suppl 1, 34 41. https://doi.org/10.1258/jhsrp.2010.010092

Nicolini, D., Waring, J., Mengis, J., 2011b. Policy and practice in the use of root cause analysis to investigate clinical adverse events: mind the gap. Soc. Sci. Med. 1982 73, 217-225. https://doi.org/10.1016/j.socscimed.2011.05.010

NOPSA, 2011. NOPSA Hazard Identification Guidance Note. National Offshore Petroleum Safety Authority, Australia.

NPSA, 2009. Root Cause Analysis Investigation Tools Contributory Factors Classification Framework. NPSA.

NPSA, 2006. Risk assessment programme Overview. National Patient Safety Agency, London.

Olsen, S., Neale, G., Schwab, K., Psaila, B., Patel, T., Chapman, E.J., Vincent, C., 2007. Hospital staff should use more than one method to detect adverse events and potential adverse events: incident reporting, pharmacist surveillance and local realtime record review may all have a place. Qual. Saf. Health Care 16, 40-44. https://doi.org/10.1136/qshc.2005.017616

Potts, H.W., Anderson, J.E., Colligan, L., Leach, P., Davis, S., Berman, J., 2014. Assessing the validity of prospective hazard analysis methods: a comparison of two techniques. BMC Health Serv. Res. 14, 41. https://doi.org/10.1186/1472-6963-14-41

Reason, J., 1990. Human Error. Cambridge University Press, Cambridge.

Redmill, F., Chudleigh, M., Catmur, J., 1999. System safety: HAZOP and software HAZOP. Wiley, Chichester; New York.

Rogers, S., 2002. A structured approach for the investigation of clinical incidents in health care: application in a general practice setting. Br. J. Gen. Pract. 52, S30-S32.

Rollenhagen, C., Westerlund, J., Lundberg, J., Hollnagel, E., 2010. The context and habits of accident investigation practices: A study of 108 Swedish investigators. Saf. Sci. 48, 859-867. https://doi.org/10.1016/j.ssci.2010.04.001

Runciman, W.B., Williamson, J.A.H., Deakin, A., Benveniste, K.A., Bannon, K., Hibbert, P.D., 2006. An integrated framework for safety, quality and risk management: an information and incident management system based on a universal patient safety classification. Qual. Saf. Health Care 15, i82-i90. https://doi.org/10.1136/qshc.2005.017467

Sammer, C.E., Lykens, K., Singh, K.P., Mains, D.A., Lackan, N.A., 2010. What is Patient Safety Culture? A Review of the Literature. J. Nurs. Scholarsh. 42, 156-165. https://doi.org/10.1111/j.1547-5069.2009.01330.x 
Sari, A.B.-A., Sheldon, T.A., Cracknell, A., Turnbull, A., 2007. Sensitivity of routine system for reporting patient safety incidents in an NHS hospital: retrospective patient case note review. BMJ 334, 79. https://doi.org/10.1136/bmj.39031.507153.AE

Senders, J., 2004. FMEA and RCA: the mantras*; of modern risk management. Qual. Saf. Health Care 13, 249-250. https://doi.org/10.1136/qshc.2004.010868

Shojania, K.G., 2012. Deaths due to medical error: jumbo jets or just small propeller planes? BMJ Qual. Saf. 21, 709-712. https://doi.org/10.1136/bmjqs-2012-001368

Shojania, K.G., Dixon-Woods, M., 2013. "Bad apples": time to redefine as a type of systems problem? BMJ Qual. Saf. 22, 528-531. https://doi.org/10.1136/bmjqs-2013002138

Simsekler, M.C.E., 2019. The Link Between Healthcare Risk Identification and Patient Safety Culture. Int. J. Health Care Qual. Assur. 32, 574-587. https://doi.org/10.1108/IJHCQA-04-2018-0098

Simsekler, M.C.E., Card, A.J., Ruggeri, K., Ward, J.R., Clarkson, P.J., 2015a. A comparison of the methods used to support risk identification for patient safety in one UK NHS foundation trust. Clin. Risk 21, 37-46. https://doi.org/10.1177/1356262215580224

Simsekler, M.C.E., Card, A.J., Ward, J.R., Clarkson, P.J., 2015b. Trust-Level Risk Identification Guidance in the NHS East of England. Int. J. Risk Saf. Med. 27, 67-76. https://doi.org/10.3233/JRS-150651

Simsekler, M.C.E., Jayaraman, R., 2018. Risk Identification Practice in Patient Safety Context. Presented at the 2018 IEEE International Conference on Industrial Engineering and Engineering Management (IEEM), IEEE, Bangkok, pp. 1756-1760. https://doi.org/10.1109/IEEM.2018.8607315

Simsekler, M.C.E., Kaya, G.K., Ward, J.R., Clarkson, P.J., 2019. Evaluating Inputs of Failure Modes and Effects Analysis in Identifying Patient Safety Risks. Int. J. Health Care Qual. Assur. 32, 191-207. https://doi.org/10.1108/IJHCQA-12-2017-0233

Simsekler, M.C.E., Ward, J.R., Clarkson, P.J., 2018a. Design for patient safety: a systems-based risk identification framework. Ergonomics 61, 1046-1064. https://doi.org/10.1080/00140139.2018.1437224

Simsekler, M.C.E., Ward, J.R., Clarkson, P.J., 2018b. Evaluation of system mapping approaches in identifying patient safety risks. Int. J. Qual. Health Care 30, 227-233. https://doi.org/10.1093/intqhe/mzx176

Smith, A., Boult, M., Woods, I., Johnson, S., 2010. Promoting patient safety through prospective risk identification: example from peri-operative care. Qual. Saf. Health Care 19, 69-73. https://doi.org/10.1136/qshc.2008.028050 
Smith, B.E., Jong, H.H. de, Everdij, M.H.C., 2008. A prognostic method to identify hazards for future aviation concepts, in: ICAS 2018. Presented at the 26th International Congress of the Aeronautical Sciences, Alaska, USA.

Spurgeon, P., Flanagan, H., Cooke, M., Sujan, M., Cross, S., Jarvis, R., 2017. Creating safer health systems: Lessons from other sectors and an account of an application in the Safer Clinical Systems programme. Health Serv. Manage. Res. 30, 85-93. https://doi.org/10.1177/0951484817696211

Sujan, M., 2015. An organisation without a memory: A qualitative study of hospital staff perceptions on reporting and organisational learning for patient safety. Reliab. Eng. Syst. Saf. 144, 45-52. https://doi.org/10.1016/j.ress.2015.07.011

Sujan, M., Spurgeon, P., Cooke, M., 2015. The role of dynamic trade-offs in creating safety-A qualitative study of handover across care boundaries in emergency care. Reliab. Eng. Syst. Saf., Special Issue on Resilience Engineering 141, 54-62. https://doi.org/10.1016/j.ress.2015.03.006

Sujan, M.A., 2012. A novel tool for organisational learning and its impact on safety culture in a hospital dispensary. Reliab. Eng. Syst. Saf. 101, 21-34. https://doi.org/10.1016/j.ress.2011.12.021

Sujan, M.A., Embrey, D., Huang, H., 2018. On the application of Human Reliability Analysis in healthcare: Opportunities and challenges. Reliab. Eng. Syst. Saf. https://doi.org/10.1016/j.ress.2018.06.017

Sujan, M.-A., Felici, M., 2012. Combining Failure Mode and Functional Resonance Analyses in Healthcare Settings, in: Ortmeier, F., Daniel, P. (Eds.), Computer Safety, Reliability, and Security, Lecture Notes in Computer Science. Springer Berlin Heidelberg, pp. 364-375.

Sujan, M.A., Habli, I., Kelly, T.P., Gühnemann, A., Pozzi, S., Johnson, C.W., 2017 a. How can health care organisations make and justify decisions about risk reduction? Lessons from a cross-industry review and a health care stakeholder consensus development process. Reliab. Eng. Syst. Saf. 161, 1-11. https://doi.org/10.1016/j.ress.2017.01.001

Sujan, M.A., Habli, I., Kelly, T.P., Pozzi, S., Johnson, C.W., 2016a. Should healthcare providers do safety cases? Lessons from a cross-industry review of safety case practices. Saf. Sci. 84, 181-189. https://doi.org/10.1016/j.ssci.2015.12.021

Sujan, M.A., Huang, H., Braithwaite, J., 2017b. Learning from incidents in health care: Critique from a Safety-II perspective. Saf. Sci., Learning from Incidents 99, 115-121. https://doi.org/10.1016/j.ssci.2016.08.005

Sujan, M.A., Pozzi, S., Valbonesi, C., 2016b. Reporting and Learning: From Extraordinary to Ordinary, in: Resilient Health Care III: Reconciling Work-AsImagined and Work-As-Done. CRC Press. https://doi.org/info:doi/10.9774/GLEAF.9781498780575_10 
Thomas Diller, George Helmrich, Sharon Dunning, Stephanie Cox, April Buchanan, Scott Shappell, 2014. The Human Factors Analysis Classification System (HFACS) Applied to Health Care. Am. J. Med. Qual. 29, 181-190. https://doi.org/10.1177/1062860613491623

van Tilburg, C.M., Leistikow, I.P., Rademaker, C.M.A., Bierings, M.B., van Dijk, A.T.H., 2006. Health care failure mode and effect analysis: a useful proactive risk analysis in a pediatric oncology ward. Qual. Saf. Health Care 15, 58-63. https://doi.org/10.1136/qshc.2005.014902

Vincent, C., 2010. Patient Safety, 2nd Edition edition. ed. Wiley-Blackwell.

Vincent, C., 2006. Patient Safety. Churchill Livingstone Elsevier, London.

Vincent, C., Taylor-Adams, S., Stanhope, N., 1998. Framework for analysing risk and safety in clinical medicine. BMJ 316, 1154-1157. https://doi.org/10.1136/bmj.316.7138.1154

Vincent, C.A., 2004. Analysis of clinical incidents: a window on the system not a search for root causes. Qual. Saf. Health Care 13, 242-243. https://doi.org/10.1136/qhc.13.4.242

Vincent, C.J., Ward, J., Langdon, P., 2012. Unravelling complex systems. The Ergonomist 506, 12-13.

Ward, J.R., Clarkson, P.J., Buckle, P., Berman, J., Lim, R., Jun, G.T., 2010. Prospective Hazard Analysis: Tailoring Prospective Methods to a Healthcare Context. Patient Safety Research Programme of the Department of Health, Cambridge.

Waterson, P., 2009. A critical review of the systems approach within patient safety research. Ergonomics 52, 1185-1195. https://doi.org/10.1080/00140130903042782

Weingart, S.N., Weissman, J.S., Zimmer, K.P., Giannini, R.C., Quigley, D.D., Hunter, L.E., Ridgely, M.S., Schneider, E.C., 2017. Implementation and evaluation of a prototype consumer reporting system for patient safety events. Int. J. Qual. Health Care 29, 521-526. https://doi.org/10.1093/intqhe/mzx060

Westbrook, J.I., Li, L., Lehnbom, E.C., Baysari, M.T., Braithwaite, J., Burke, R., Conn, C., Day, R.O., 2015. What are incident reports telling us? A comparative study at two Australian hospitals of medication errors identified at audit, detected by staff and reported to an incident system. Int. J. Qual. Health Care 27, 1-9. https://doi.org/10.1093/intqhe/mzu098

Woloshynowych, M., Rogers, S., Taylor-Adams, S., Vincent, C., 2005. The investigation and analysis of critical incidents and adverse events in healthcare. Health Technol. Assess. Winch. Engl. 9, 1-143, iii. 


\section{Tables}

\begin{tabular}{|c|c|c|}
\hline & Reactive Risk Identification Methods & Proactive Risk Identification Methods \\
\hline Definition & - Identify risks after safety events occur & $\begin{array}{l}\text { - Identify safety concerns and hazards } \\
\text { before they occur }\end{array}$ \\
\hline $\begin{array}{l}\text { When to } \\
\text { use }\end{array}$ & - In response to safety events & $\begin{array}{l}\text { - To identify potential risks before they } \\
\text { occur }\end{array}$ \\
\hline Strengths & $\begin{array}{l}\text { - Help learn from past experiences } \\
\text { - Identify root cause and contributory } \\
\text { factors (via RCA) } \\
\text { - Provide benchmarkable dataset on past } \\
\text { risks }\end{array}$ & $\begin{array}{l}\text { - Proactive } \\
\text { - Mainly based on systems approaches } \\
\text { - Learn from many events } \\
\text { - Considering past, present and future } \\
\text { prediction } \\
\text { - Help prioritize resources } \\
\text { - Help redesign processes to improve } \\
\text { - Mafety } \\
\text { - More open and extensive compared to } \\
\text { reactive methods } \\
\text { - Availability of various methods to meet } \\
\text { particular needs of the risk identification } \\
\text { imagining of new risks } \\
\text { imaginatinstorming facilitates the }\end{array}$ \\
\hline Weaknesses & $\begin{array}{l}\text { - Reactive nature } \\
\text { - Analyse only a single event } \\
\text { - Underreporting } \\
\text { - Incomplete data } \\
\text { - Hindsight bias } \\
\text { - Unreliable classifications in reported } \\
\text { incidents } \\
\text { - Time delay between reporting and data } \\
\text { feedback to hospitals } \\
\text { - Little evidence of measurable and } \\
\text { sustainable improvements in the area } \\
\text { of patient safety } \\
\text { - In reporting, only serious incidents are } \\
\text { generally reported, but not near misses } \\
\text { or potential errors } \\
\text { - Lack of time and expertise and } \\
\text { problems translating RCA recommen- } \\
\text { dations into tangible service changes } \\
\text { - Difficulty in converting learning points } \\
\text { from root cause analysis into } \\
\text { appropriate risk treatment plans }\end{array}$ & $\begin{array}{l}\text { - Lack of expertise in healthcare } \\
\text { - Lack of training } \\
\text { - Time-intensive } \\
\text { - Little evidence on their reliability and } \\
\text { validity in healthcare practice } \\
\text { - Very slow and sporadic adoption of PHA } \\
\text { methods in healthcare } \\
\text { - The variety of methods makes it difficult } \\
\text { to choose the most suitable method for a } \\
\text { particular system assessed } \\
\text { - Structured brainstorming in some } \\
\text { methods hinder the imagining of new } \\
\text { risks }\end{array}$ \\
\hline
\end{tabular}

Table 1 Comparison of reactive versus proactive risk identification approaches 


\section{Figures}

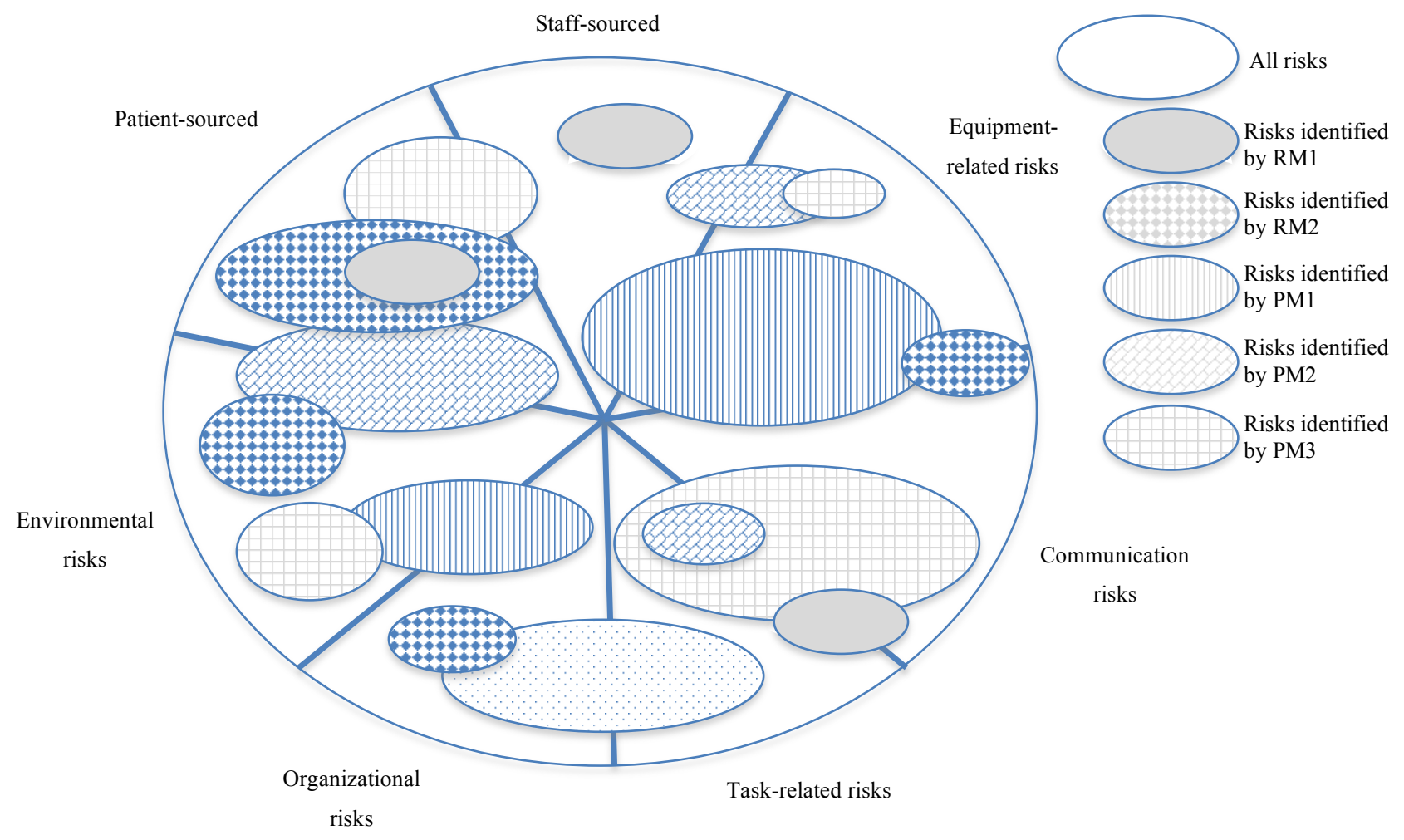

Figure 1 Integration of all risk information in one picture [As depicted in Figure 1, RM1 and RM2 denote reactive methods, while PM1, PM2 and PM3 are proactive methods. Despite some inevitable overlap between risks identified by the respective methods, each method may identify a new risk and add value to the overall risk identification practice.]

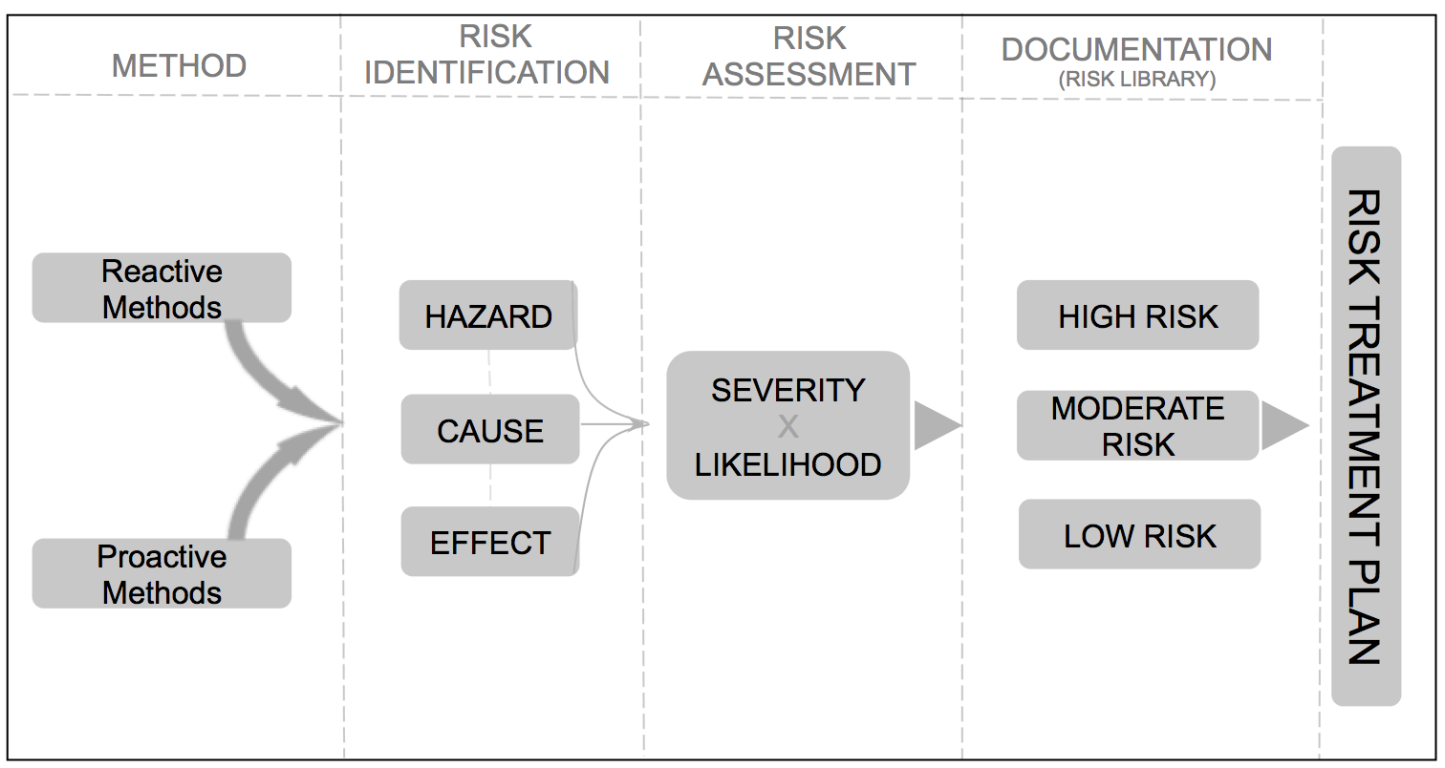

Figure 2 Risk Library in a Risk Management Process 